\title{
Negotiating The Maze: Confronting Dysphagia Together With My Stroke-Afflicted Family Member
}

Ying-Jung Tseng, RN, MSN, PhD Candidate

School of Nursing, National Taipei University of Nursing and Health Sciences and Chang-Gung University

Science and Technology, Taiwan (ROC)

Lee-Ing Tsao, RN, MSN, DNS

College of Nursing \& Vice President, National Taipei University of Nursing and Health Sciences, Taiwan (ROC)

\section{Hsueh-Pei Wang, MSN}

Stroke and Brain Injury Rehabilitation Center, Endeavor Rehabilitation Clinic, Taiwan (ROC)

\section{Vinchi Wang, MD, PhD}

Department of Neurology, Cardinal Tien Hospital, New Taipei City and School of Medicine, College of Medicine, Fu-Jen Catholic University, Taiwan (ROC)

\section{Mei-Hui Chen, RN, BSN}

Department of Physical Medicine and Rehabilitation, Chang Gung Memorial Hospital, Taiwan (ROC)

\section{Chin-Man Wang, MD}

Department of Physical Medicine and Rehabilitation, Chang Gung Memorial Hospital and Department of Medicine,

Chang Gung University, Taiwan (ROC)

\section{Shu-Li Lin, RN, BSN}

Department of Nursing, Chang Gung Memorial Hospital, Taiwan (ROC)

\section{Yu-Li Lin, RN, MSN}

Department of Nursing, Chang Gung Memorial Hospital,

Taoyuan and Department of Nursing,

Chang Gung University Science and Technology, Taiwan (ROC)

\section{doi: 10.19044/esj.2016.v12n24p63 URL:http://dx.doi.org/10.19044/esj.2016.v12n24p63}

\begin{abstract}
Aim: To generate a descriptive theory grounded in the responses of family caregivers caring for their family stroke survivors with dysphagia during hospitalization. Design and Method: A qualitative study employing the grounded theory method was used. Fifteen family caregivers participated
\end{abstract}


in comprehensive interviews. The interview data were analyzed using the constant comparative method. Findings: 'Negotiating the maze: Confronting dysphagia with my stroke-afflicted family member' was the core category guiding the care process for dysphagia family members among caregivers. After surviving stroke, the caregivers felt 'more confusion less rejoicing' as the antecedent condition. The following three interaction categories were identified: (1) 'being overwhelmed by nasogastric (NG) tube issues'; (2) 'searching for the right helper and information'; and (3) 'food culture conflicts with the formula diet administered through the NG tube'. Additionally, 'Maintaining positivity' described the consequence of this process. Conclusions: This study highlights the critical perspective of family members who care for dysphagia stroke survivors in the hospital. Participants were under tremendous pressure during the disease treatment process. However, all attempted to maintain a positive attitude and treasured the chance to accompany their family members. Clinical Relevance: These findings can assist health professionals in charting the effects of dysphagia and in understanding the problems and needs according to the subjective perspectives of family caregivers. They can also provide a necessary foundation for comprehensive care interventions for family caregivers of stroke survivors with dysphagia.

Keywords: Stroke, dysphagia, family caregivers, grounded theory

\section{Introduction}

Stroke is the third leading cause of death and the most common cause of complex disability in Taiwan (Hsieh \& Chiou, 2014). Approximately 50 to $55 \%$ of acute stroke survivors are affected by dysphagia (Dziewas et al., 2008; Martino et al., 2005), which has been identified as an independent predictor of mortality and an important risk factor for dehydration, malnutrition and aspiration pneumonia in stroke survivors (Martino et al., 2005; Rosenvinge \& Starke, 2005; Smithard et al., 1996). Post-stroke dysphagia usually spontaneously recovers within 14 days; however, up to $7 \%$ of stroke survivors remain dysphagic at 3 months (Martino, Pron \& Diamant, 2000; Smithard et al., 1997).

Family caregivers are important members of support teams for people with dysphagia (Nund, Scarinci, Cartmill \& Ward, 2015). However, family caregivers feel a heavy burden, especially in relation to food and meal preparation, when caring for dysphagia stroke survivors at home (Arai, 2005; Choi-Kwon, Kim, Kwon \& Kim, 2005; Johansson \& Johansson, 2009; Nund et al., 2015). Less research has been conducted on the effects of dysphagia among family caregivers of stroke survivors during the stroke recovery process in the hospital (Martino, Beaton \& Diamant, 2009; Nund et al., 
2015). The aim of this study was to generate a descriptive theory based on the life experiences of family caregivers who stayed with dysphagic stroke relatives during hospitalization.

\section{Methods}

This qualitative study employed a grounded theory that was described by Corbin and Strauss $(1990,2014)$ encompassing several unique methodological elements, such as constant comparative analysis and theoretical sampling, to develop a new "theory" that is grounded in empirical data from the field (Corbin \& Strauss, 2014; Dunne, 2011).

\section{Sampling and ethical consideration}

Purposive and snowball sampling techniques were conducted to identify the participants in this study. The inclusion criteria were: (1) at least 20 years of age, (2) provided stroke patients with unpaid help for more than 3 days per week and the stroke diagnosis was confirmed with a physician's clinical note as well as a CT scan or MRI findings, (3) willing to share their experiences in caring for patients who had undergone or were currently undergoing speech therapy to treat dysphagia, (4) able to speak Taiwanese or Mandarin, and (5) willing to provide written informed consent.

This study was approved by the institutional review board of an academic medical center in Taiwan (No. 100-0486B). Participation was voluntary, and all of the participants were guaranteed strict confidentiality and anonymity. The participants were allowed to withdraw from the study at any time without penalty.

\section{Data collection and analysis}

The study spanned from December 2010 to February 2012. Fifteen subjects were recruited to participate in the interviews and data collection. All interviews were audio recorded. The interviews averaged 60 minutes in length. The interviewer followed semi-structured interview guidelines, which included the following questions. (1) How did you know your relative had difficulty swallowing? (2) What do you do to alleviate your relative's swallowing difficulties? (3) What types of issues do you encounter when you take care of your dysphagic relative? (4) What are your needs when you take care of your dysphagic relative?

Every interview was analyzed immediately to identify ideas. The interview transcripts were coded with a qualitative software program called ATLAS.ti (version 6.2.28, ATLAS.ti Scientific Software Development $\mathrm{GmbH}$, Berlin, Germany, 2012). Interviews were continued until the data were saturated and dense. 
The following four criteria were applied to evaluate the rigor of this study: (1) credibility, (2) dependability, (3) conformability, and (4) transferability (Guba \& Lincoln, 1994). With respect to credibility, the first author has cared for stroke patients and family caregivers for more than 20 years, has researched dysphagia issues for more than 6 years and has established a trusting relationship with some of the participants by accompanying them and their stroke relatives to swallowing training classes. Therefore, the participants were willing to share their subjective experiences in greater detail. Professional health referral and snowball sampling methods were used to increase the acceptance of the participants. Additionally, the researcher used open-ended interview questions, verified participant responses and asked the participants to validate the findings, which resulted in increased data credibility. The first author (YJT) and an experienced mentor (LYC) were involved in the data collection and analysis, and they constantly compared and recorded the transcripts to ensure the reliability of this study. Conformability was established by recruiting participants who were willing to share their experiences, followed by the review, discussion, critique and confirmation of the coding and the categorization of the data by the authors. Finally, transferability was established by recruiting participants from various backgrounds to enable the researcher to obtain sufficient information from the participants to apply these findings to other family caregivers of stroke survivors.

\section{Results}

The patients were sampled from the rehabilitation departments of three hospitals in northern Taiwan, which included an academic medical center, a metropolitan hospital and a community rehabilitation hospital. Fifteen caregivers participated in this study, including 10 spouses (66.7\%), 4 children (26.7\%) and 1 sister-in-law. The mean participant age was 55 years (ranging from 32 to 81). The mean age of their stroke patient relatives was 65 years (ranging from 40 to 86). The mean length of NG tube use was 190 days (ranging from 28 to 600 days). The family caregivers reported that the mean length of speech therapy for their stroke patient relatives was 106 days (ranging from 3 to 540 days) (details listed in Table 1).

\section{Theoretical framework}

The theoretical framework of this study is shown in Figure 1. "Negotiating the maze: Confronting dysphagia with my stroke-afflicted family member" was the core category for addressing swallowing issues when family caregivers took care of stroke survivors with dysphagia as the patients progressed through the stroke recovery processes in the hospital. 
Table 1. Family Caregiver Demographic Data $(\mathrm{N}=15)$

\begin{tabular}{ccc}
\hline Characteristics & N (\%) & Range (mean) \\
\hline Age (years) & & \\
Gender & $10(66.7)$ & \\
Female & $5(33.3)$ & \\
Male & & \\
Relationship to patient & $4(26.7)$ & \\
Children & $10(66.7)$ & \\
Spouses & $1(6.6)$ & \\
Sister-in-law & & \\
Marital status & $3(20.0)$ & \\
Single & $12(80.0)$ & \\
Married & & \\
Education level & $3(20.0)$ & \\
University & $6(40.0)$ & \\
Junior college & $2(13.3)$ & \\
Vocational high school & $3(20.0)$ & \\
Middle school & $1(6.7)$ & \\
Primary school & & \\
Occupation & $1(6.7)$ & \\
Unemployed & $5(33.3)$ & \\
Business & $2(13.3)$ & \\
Industry & $4(26.7)$ & \\
Housewife & $3(20.0)$ & \\
Retired & & \\
Age of patient (years) & & \\
Duration from stroke onset (days) & & \\
Duration of dysphagia (days) & & \\
Duration of speech therapy (days) & & \\
\hline & & \\
\hline
\end{tabular}

"More confusion less rejoicing" was identified as the antecedent condition. From the acute stage to the rehabilitation stage of the stroke treatment process, family caregivers met three major issues, which were categorized as "Being overwhelmed by NG tube issues," "Searching for right helpers and information," and "Food culture conflicts with the formula diet administered through the NG tube." Whatever choice the family caregivers made, they tried to be positive to obtain appropriate assistance that would help them move towards a positive result. "Maintaining positivity" described the consequence of this process. 
Figure 1. Negotiating the maze: Confronting dysphagia together with my stroke-afflicted

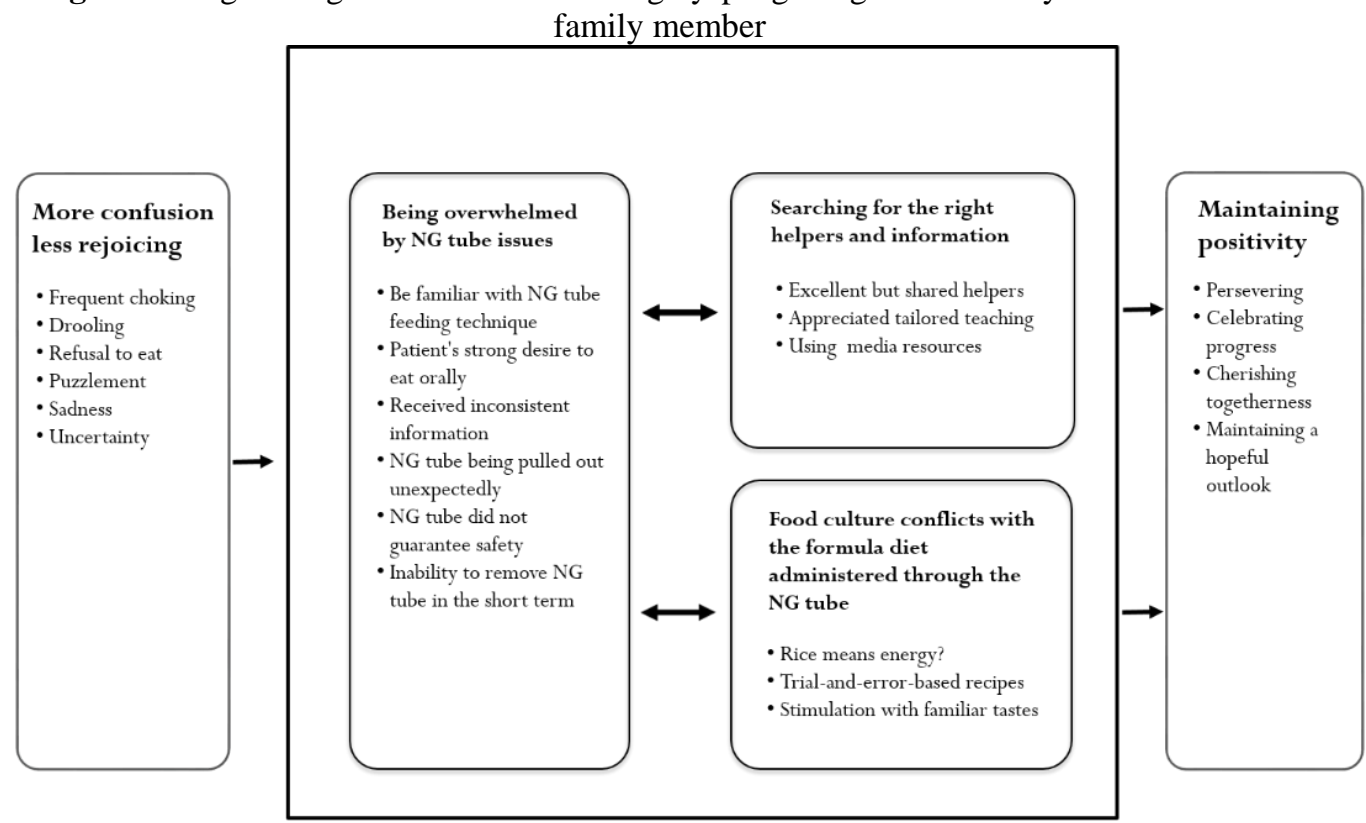

\section{Negotiating the maze: Confronting dysphagia together with my stroke- afflicted family member}

Family members accompanying stroke patients with dysphagia faced challenging experiences that were likened to walking in a maze with the need to find an exit as soon as possible. Most family caregivers did not understand the meaning of "dysphagia” prior to their relative's stroke; however, they came to understand the dangers of dysphagia as their relative's hospital stay progressed. Although these participants were unsure of the future, they attempted to accompany, protect and help their relatives while maintaining positive thinking when confronting challenges during the recovery process.

\section{More confusion less rejoicing}

After the patients survived stroke, their caregivers felt "more confusion less rejoicing". The sequence of events they met post-stroke produced puzzlement, sadness and uncertainty. They said that they did not know that frequent choking, drooling and refusal to eat usually indicates that a stroke survivor has dysphagia. Additionally, "refusal to eat" was the strangest and most painful experience for the family caregivers. The majority of family caregivers did not realize that dysphagia is a life-threatening situation, and they could not accept that patients with dysphagia frequently required tube feeding. A 71-year-old wife recounted tearfully, "At that time, he refused to eat. I tried many kinds of food to make him eat... finally, they said he got aspiration pneumonia. He was on antibiotics for a long time. 
Then, I realized I should not have let him eat at the beginning. It was me... I let him suffer like this... However, at that time, no one told me I should stop feeding him...”. (Case 5)

\section{Being overwhelmed by NG tube issues}

Family caregivers faced an unprecedented plight when their strokeafflicted relatives were tube-fed. They were met with several issues: familiarity with the NG tube feeding technique, the patient's strong desire to eat orally, receiving inconsistent information, the NG tube being pulled out unexpectedly, the NG tube not guaranteeing safety and the inability to remove the NG tube in the short term.

The participants were frightened to hear that they should learn tube feeding skills. If they could not take care of their relatives, particularly regarding NG tube feeding, they would hire a nursing assistant to help them. As a wife said, "The first day in the emergency room, my husband just had his NG tube inserted, and a nurse came and pushed me to learn how to perform NG feeding. I was so shocked; I was 80 years old... I was frightened to do NG feeding. (Case 2)

When the stroke survivors repeatedly expressed hunger and continuously asked to eat orally, their family caregivers felt great sadness. Additionally, the majority of participants said that a change in position or sneezing may cause the NG tube to come out unexpectedly, and a tube that had been clogged without any apparent reason sometimes led to the need for a new NG tube insertion.

Family caregivers described that they received inconsistent information from many sources, including physicians, nurses, other family members or nursing assistants. Some found that physicians and nurses from the neurology and rehabilitation departments provided contradicting information. The health professionals in the rehabilitation department were stricter. Within the same department, different nurses had different suggestions regarding the same issue. One husband said, "We started to practice eating orally in the neurology department on the doctor's advice. However, on being transferred to the rehabilitation department, doctors and nurses strictly prohibited us from eating orally. Now we were in the rehabilitation department, we had better to follow their rules. We performed oral feeding in secret." (Case 4)

Most participants regarded the NG tube as a protective safety device. They were shocked that their relatives suddenly developed aspiration pneumonia when using the NG tube. Moreover, family caregivers did not understand why it was so difficult to remove an NG tube. This situation caused some participants to be doubted by other family members. One participant said, "Taking care of my husband was not the biggest task; his 
elderly relatives were. Every time they came to see him, they all criticized me; why couldn't he remove the NG tube after such a long time? They told me, "You better train him orally", and "You better..." They doubted my ability...Every time they left, I would cry. I felt traumatized by their visits." (Case 11)

\section{Searching for the right helper and information}

During the stroke treatment process, family caregivers received conflicting ideas from others. They expected to find available, accountable, experienced experts and were eager to receive correct and useful information. Most participants felt substantial support from the SLTs. The SLTs provided tailored teaching, including concrete tips for stimulating the swallowing reflex, tongue exercises, face massage, protection methods and eating strategies. Unfortunately, there are too few SLTs in Taiwan, and the families had to wait for an extended period to be assigned one. The participants found that nurses and doctors were too busy and seldom gave dysphagia-related instructions in great detail, such as procedures for oral care, feeding, food preparation or addressing problems during swallowing practice. A participant said, "My brother searched on Google, and he found this hospital and gave me the message. Why did I choose this non-insurancesupported hospital (the community rehabilitation hospital)? Because the doctors in the rehabilitation department in the other insurance-supported hospitals couldn't take in my husband. They said he was too drowsy, so he

couldn't do any training. But, I knew he was conscious at certain times of day and I believed he can learn something." (Case 14)

\section{Food culture conflicts with the formula diet administered through the NG tube}

Most family caregivers believed that "rice means energy," as is prevalent in Chinese food culture philosophy; they attempted to use trialand-error-based recipes with familiar tastes to help their dysphagic relatives recover quickly. The participants strongly suspected that the flavorless NG diet contained fewer nutrients than normal food, particularly rice. Therefore, in addition to following the instructions of the health professionals to feed their relatives via an NG tube, they also used alternative strategies to increase the stroke survivors' energy. As one wife said, "In order to increase his energy, I insisted on feeding him 7 bottles of a formula diet in a day. In using the NG tube, I could feed him at any time, and he would not choke in his sleep. I would prepare porridge or rice for lunch, when he was most alert, and he could eat by himself." (Case 11)

All participants used trial-and-error-based recipes. They admitted that they gave some food to their relatives during treatment despite eating orally 
was banned by the health professionals. The strongest reason for these efforts was that they could not tolerate it when their beloved relatives expressed hunger and asked for something to eat. Some of the caregivers thought that giving the patients a chance to eat simply meant that they had a chance to help the patients. When the patients succeeded in eating something, the family caregivers felt some sense of achievement and continued to feed the patients orally in secret. The patients would sometimes severely choke, and the family caregivers would stop the training for a while. As one participant stated, "Eating orally is a natural ability. Look at him; he could not eat anything for half a year. We did not know what we could do. So we decided to give something to him just to train his eating ability. We hoped that it would help him have good progress one day." (Case 5) Similarly, a patient's sister-in law stated, "I wanted her to eat some porridge to improve her energy. There's a kind of ancient Chinese wisdom. It says, 'Rice or porridge can increase physical energy.' I found it useful. I cooked a delicious porridge for her. The recipe included bone broth, ground meat, diced whitebait, onions and carrots. Everything was cooked together until it became a thick porridge. Sometimes I cooked fish soup. I let her eat the meat orally and try some soup. If she choked, I would stop and feed it to her through the NG tube. (Case 13)

Some participants used familiar items to stimulate stroke patients and to attempt to speed up the rehabilitation process. As one husband said, "Someone told me I could stimulate my wife with something she loved. I thought she loved coffee, so I gave it to her. When she took the first sip of coffee, I saw a big smile on her face, just so satisfied. I trained her from that time. I gave her everything she loved, including cherries." (Case 8) Another participant noted, "I was bolder than others; I bought a Mister Donut for him in the beginning. I tried to use something he loved to stimulate him. If he couldn't swallow it all, I would dig out the leftover food. I think it worked." (Case 14)

\section{Maintaining positivity}

Family caregivers in this study experienced challenges in accompanying their stroke-afflicted relatives from the acute stage to the rehabilitation stage; however, they persevered, celebrated progress, cherished togetherness and remained hopeful for the future. They hoped that the patients would be able to have the NG tube removed and eat food orally as soon as possible. The caregivers helped their relatives set an easily reachable goal, encouraging them to move forward with hope and creating an enjoyable and safe environment even if some of patients would not go without the feeding tube for the rest of their lives. As one son said, "I did not care if she recovered to normal or not. I tried to arrange her lifestyle, 
including continuing some rehabilitation training and getting outside. I hoped she would have a good quality of life and not just lie down on the bed like a 'real' patient. I hoped we could continue in this direction. Now, 1.5 years have passed, and everything went pretty nicely! My mom is used to this lifestyle." (Case 7)

\section{Discussion}

In Taiwan, most hospitalized patients have relatives, foreign laborers or nursing assistants with them 24 hours a day (Yeh \& Chang, 2011). In the current study, we found that family caregivers played an important role throughout the post-stroke hospitalization process. These caregivers assisted their dysphagic relatives with several daily activities, including feeding them via NG tube, accompanying them to dysphagia treatment classes and following the instructions of the speech-language therapists (SLTs) to practice swallowing. The current results differ from those of other studies, which focus on the role changes of family caregivers of dysphagic stroke patients on home-based responsibilities (Arai, 2005; Cameron, Naglie, Silver \& Gignac, 2012; Johansson \& Johansson, 2009; Nund et al., 2015; Wallengren, Friberg \& Segesten, 2008). The data regarding the roles or life experiences of family caregivers of dysphagic stroke patients during hospitalization were limited.

The majority of family caregivers in this study failed to notice the indicators of dysphagia, especially in the acute stage. This finding was consistent with the study conducted by Cohen et al. (2016). They noted that patients and their relatives are often unaware that a stroke can cause swallowing problems (Cohen et al., 2016). Some participants did not regard the signs and symptoms of dysphagia, such as choking, drooling or refusing to eat, as a serious matter and continued to feed the patients orally. These findings indicate that the early identification of dysphagia by nurses and appropriate referrals of patients to SLTs for further assessment are paramount (Malhi, 2016). Moreover, family caregivers should receive information regarding the after-effects of a stroke as soon as possible (Garrett \& Cowdell, 2005).

Early tube feeding is associated with a reduction in the risk of death in dysphagic stroke patients (Dennis, Lewis \& Warlow, 2005; Rowat, 2015). In this study, all participants complained that NG tubes extremely troublesome. Some were frightened upon seeing relatives who had developed aspiration pneumonia during NG tube use. This finding is similar to previous research, which presents several adverse events during the use of a nasal tube, such as the tube being easily dislodged, nasopharyngeal discomfort, chronic sinusitis, gastroesophageal reflux, and aspiration pneumonia (Brogan, Langdon, Brookes, Budgeon \& Blacker, 2014; Rowat, 2015). 
Approximately 38 to $43 \%$ of NG-tube-fed stroke survivors develop aspiration pneumonia (Brogan et al., 2014; Ciocon, Silverstone, Graver \& Foley, 1988; Gomes et al., 2015). Some risk factors may be related to aspiration pneumonia of stroke patients in our study, such as poor oral care, lack of elevation while feeding, or being fed orally in secret.

NG tube feeding and percutaneous endoscopic gastrostomy (PEG) are commonly used for stroke patients who cannot swallow (Rowat, 2015). NG tube feeding is the preferred method for short-term feeding and a PEG tube is used for feeding stroke patients who require longer-term nutritional support (Dennis et al., 2005; Gomes et al., 2015; Rowat, 2015). Furthermore, PEG was associated with a lower likelihood of intervention failure, less inconvenience, discomfort and altered body image and was more effective and safe compared with an NG tube (Gomes et al., 2015). In our study, all stroke patients used NG tube feeding throughout their long-term hospitalization. Further research regarding the reasons for the long-term use of NG tubes and acceptance of PEG placement by patients and their families in Taiwan may be required.

A multidisciplinary team approach offers an effective model for the management of post-stroke dysphagia (Aoki et al., 2016; Gandolfi et al., 2014). A multidisciplinary team may consist of neurologists, nurses, rehabilitation physicians, speech therapists, physical therapists, nutritionists, radiologists, and pharmacists (Aoki et al., 2016; Gandolfi et al., 2014). The family caregivers in the present study were unsatisfied with the lack of adequate information regarding dysphagia care, especially from physicians and nurses. They strongly recommended that health professionals should support them and their stroke-afflicted relatives with a positive attitude; in the early post-stroke stage, these family members should be provided with detailed dysphagia care information on disease prognosis, predicted recovery, treatment plans, care tips, precautions and any available resources. Additionally, the participants in our study appreciated the dysphagia treatment conducted by SLTs; however, most complained about the long wait list for an SLT. This result is echoed in the survey conducted by Chen et al. (2007), who reported that there is a large shortage of SLTs in Taiwan. These findings may indicate the lack of a standardized diagnostic and rehabilitative multidisciplinary dysphagia protocol in Taiwanese hospitals.

Throughout Chinese history, porridge has been served as a meal and is said to preserve good health (Hsueh \& Hu, 2008; Su, 2012). Congee (rice porridge) is especially popular among Taiwanese (Su, 2012; Tzeng, Kao, Yeh \& Pan, 1999). Family caregivers in this study expressed the beliefs that "eating orally is better than tube feeding" and "rice means energy." Therefore, they fed their relatives congee or another rice product orally in secret. This result partly corresponds with the study of Nund et al, in which 
the family caregivers used trial-and-error strategies to determine which foods their dysphagic relatives could or could not eat (Nund et al., 2015). However, this behavior resulted in a risk of choking, suffocation or aspiration pneumonia. For patient safety, healthcare professionals should be aware of red flag symptoms of oral feeding.

Family caregivers in the present study experienced emotional ups and downs in parallel with the progress of their stroke-afflicted relatives during hospitalization. The participants showed that they not only felt burdens, uncertainty and sadness but that also appreciation and hope. They had positive feelings throughout the disease treatment process. The results of the present study are similar to those in the study conducted by Mackenzie and Greenwood (2012), who found that caregivers were able to identify positive experiences of caregiving that provided a more balanced view of their role.

\section{Study limitations}

This study only presented the life experiences of family caregivers for dysphagic stroke survivors who were hospitalized in the rehabilitation departments in three hospitals of northern Taiwan. Family caregivers taking care of stroke patients in other areas may encounter different issues. Data were collected during one interview; some of the caregivers needed to recall the remote experiences of the acute stages after stroke onset, and some took turns in caregiving with another family member, which may raise the issue of recall bias. Moreover, most caregivers in our study were able to participate because they hired nursing assistants to help them or because the condition of their relatives was stable; therefore, the findings may neglect other issues experienced by family caregivers who were unable to participated and therefore express their opinions.

\section{Conclusions}

This is the first study to document the life experiences of family caregivers among dysphagic stroke survivors who experienced a long period of hospitalization in Taiwan. The participants failed to notice dysphagia symptoms in the acute stage. They faced three major issues related to dysphagia during disease treatment, including NG tube feeding, searching for reliable information and assistance, and conflicts between medical orders and Chinese food culture. Although the family caregivers were under tremendous pressure, they attempted to maintain a positive attitude and treasured the opportunity to accompany their family members.

\section{Implications for clinical practice}

We have some suggestions based on the results of this study. First, healthcare professionals in Taiwanese hospitals should build a standardized 
multidisciplinary dysphagia protocol, recognize the dysphagia problems of stroke survivors and immediately refer those with suspected dysphagia to experts. Second, assessing family members’ caregiving ability and providing comprehensive, explicit and tailored information related to post-stroke dysphagia issues for family caregivers from health professionals and other accessible resources would be significantly beneficial. Third, psychological and emotional support and addressing the traditional Chinese food culture philosophy during post-stroke hospitalization are necessary. The findings in this study can guide healthcare professionals in providing a more holistic, continuous and family-centered approach to provide the best support and resources to meet the needs of stroke survivors and their family caregivers.

\section{References:}

Aoki, S., Hosomi, N., Hirayama, J., Nakamori, M., Yoshikawa, M., Nezu, T., ... Matsumoto, M. (2016). The multidisciplinary swallowing team approach decreases pneumonia onset in acute stroke patients. PLoS One, 11(5), e0154608. doi: 10.1371/journal.pone.0154608

Arai, K. (2005). Experiences of family caregivers for patients suffering from dysphagia--the attempt of subjective caregiving. Gan to Kagaku Ryoho. Cancer \& Chemotherapy, 32(Suppl 1), 50-52.

Brogan, E., Langdon, C., Brookes, K., Budgeon, C., \& Blacker, D. (2014). Respiratory infections in acute stroke: Nasogastric tubes and immobility are stronger predictors than dysphagia. Dysphagia, 29(3), 340-345. doi: 10.1007/s00455-013-9514-5

Cameron, J. I., Naglie, G., Silver, F. L., \& Gignac, M. A. M. (2012). Stroke family caregivers' support needs change across the care continuum: A qualitative study using the timing it right framework. Disability and Rehabilitation, 35(4), 315-324. doi: 10.3109/09638288.2012.691937

Chen, S., Yang, M., Wang, N., Li, C., Chumg, Y., Liu, S., ... Chang, C. (2007). The research of manpower supply and needs for speech pathology and audiology. Journal of the Speech-Language-Hearing Association of Taiwan, 20, 8-37.

Choi-Kwon, S., Kim, H.-S., Kwon, S. U., \& Kim, J. S. (2005). Factors affecting the burden on caregivers of stroke survivors in South Korea. Archives of Physical Medicine and Rehabilitation, 86(5), 1043-1048. doi: 10.1016/j.apmr.2004.09.013

Ciocon, J., Silverstone, F., Graver, L., \& Foley, C. (1988). Tube feedings in elderly patients. Indications, benefits, and complications. Archives of Internal Medicine, 148(2), 429-433. doi: 10.1001/archinte.148.2.429

Cohen, D. L., Roffe, C., Beavan, J., Blackett, B., Fairfield, C. A., Hamdy, S., ... Bath, P. M. (2016). Post-stroke dysphagia: A review and design 
considerations for future trials. International Journal of Stroke, 11(4), 399411. doi: 10.1177/1747493016639057

Corbin, J. M., \& Strauss, A. (1990). Grounded theory research: Procedures, canons, and evaluative criteria. Qual Sociol, 13(1), 3-21. doi: 10.1007/bf00988593

Corbin, J., \& Strauss, A. (2014). Basics of qualitative research: Techniques and procedures for developing grounded theory. Los Angeles: SAGE Publications.

Dennis, M. S., Lewis, S. C., \& Warlow, C. (2005). Effect of timing and method of enteral tube feeding for dysphagic stroke patients (FOOD): A multicentre randomised controlled trial. The Lancet, 365(9461), 764-772. doi: 10.1016/s0140-6736(05)70999-5

Dunne, C. (2011). The place of the literature review in grounded theory research. International Journal of Social Research Methodology, 14(2), 111124. doi: 10.1080/13645579.2010.494930

Dziewas, R., Warnecke, T., Hamacher, C., Oelenberg, S., Teismann, I., Kraemer, C., ... Schaebitz, W. R. (2008). Do nasogastric tubes worsen dysphagia in patients with acute stroke? BMC Neurology, 8(1). doi: 10.1186/1471-2377-8-28.

Gandolfi, M., Smania, N., Bisoffi, G., Squaquara, T., Zuccher, P., \& Mazzucco, S. (2014). Improving post-stroke dysphagia outcomes through a standardized and multidisciplinary protocol: An exploratory cohort study. Dysphagia, 29(6), 704-712. doi: 10.1007/s00455-014-9565-2

Garrett, D., \& Cowdell, F. (2005). Information needs of patients and carers following stroke. Nursing Older People, 17(6), 14-16. doi: 10.7748/nop2005.09.17.6.14.c2386

Gomes, C. A., Jr., Andriolo, R. B., Bennett, C., Lustosa, S. A., Matos, D., Waisberg, D. R., \& Waisberg, J. (2015). Percutaneous endoscopic gastrostomy versus nasogastric tube feeding for adults with swallowing disturbances. The Cochrane Database Systematic Reviews, (5), Cd008096. doi: 10.1002/14651858.CD008096.pub4

Guba, E., \& Lincoln, Y. (1994). Competing paradigms in qualitative research. In N. K. Denzin \& Y. S. Lincoln (Eds.), Handbook of qualitative research (pp. 163-194). Thousand Oaks: Sage Publications.

Hsieh, F.-I., \& Chiou, H.-Y. (2014). Stroke: Morbidity, risk factors, and care in Taiwan. Journal of Stroke, 16(2), 59-64. doi: 10.5853/jos.2014.16.2.59

Hsueh, L., \& Hu, S. (2008). When literal congee meets living congee--an introduction of Kao Lien's Tsun Sheng Pa-Ch'ien diet control. Journal of Ching Kuo Institute of Management and Health, 26, 1-19.

Johansson, A. E. M., \& Johansson, U. (2009). Relatives' experiences of family members' eating difficulties. Scandinavian Journal of Occupational Therapy, 16(1), 25-32. doi: 10.1080/11038120802257195 
Mackenzie, A., \& Greenwood, N. (2012). Positive experiences of caregiving in stroke: A systematic review. Disability and Rehabilitation, 34(17), 14131422. doi: 10.3109/09638288.2011.650307

Malhi, H. (2016). Dysphagia: Warning signs and management. British Journal of Nursing, 25(10), 546-549. doi: 10.12968/bjon.2016.25.10.546

Martino, R., Beaton, D., \& Diamant, N. E. (2009). Using different perspectives to generate items for a new scale measuring medical outcomes of dysphagia (MOD). Journal of Clinical Epidemiology, 62(5), 518-526. doi: 10.1016/j.jclinepi.2008.05.007

Martino, R., Foley, N., Bhogal, S., Diamant, N., Speechley, M., \& Teasell, R. (2005). Dysphagia after stroke: Incidence, diagnosis, and pulmonary complications. Stroke, 36(12), 2756-2763. doi: 10.1161/01.str.0000190056.76543.eb

Martino, R., Pron, G., \& Diamant, N. (2000). Screening for oropharyngeal dysphagia in stroke: Insufficient evidence for guidelines. Dysphagia, 15(1), 19-30. doi: 10.1007/s004559910006

Nund, R. L., Scarinci, N. A., Cartmill, B., \& Ward, E. C. (2015). Dysphagia and the family. In R. Speyer \& H. Bogaardt (Eds.), Seminars in dysphagia (pp. 159-175). Rijeka, Croatia: InTech.

Rosenvinge, S., \& Starke, I. (2005). Improving care for patients with dysphagia. Age and Ageing, 34(6), 587-593. doi: 10.1093/ageing/afi187

Rowat, A. (2015). Enteral tube feeding for dysphagic stroke patients. British Journal of Nursing, 24(3), 138-145. doi: 10.12968/bjon.2015.24.3.138

Smithard, D. G., O'Neill, P. A., England, R. E., Park, C. L., Wyatt, R., Martin, D. F., \& Morris, J. (1997). The natural history of dysphagia following a stroke. Dysphagia, 12(4), 188-193. doi: 10.1007/pl00009535

Smithard, D. G., O'Neill, P. A., Park, C., Morris, J., Wyatt, R., England, R., \& Martin, D. F. (1996). Complications and outcome after acute stroke: Does dysphagia matter? Stroke, 27(7), 1200-1204. doi: 10.1161/01.str.27.7.1200

$\mathrm{Su}, \mathrm{C}$. (2012). The historical perspective of Taiwan diet culture in "congee eating”. Journal of Chinese Documentation and Folk Arts, 21(1), 191-212.

Tzeng, M., Kao, M., Yeh, W., \& Pan, W. (1999). Food consumption frequency and eating habit among Taiwanese-NAHSIT 1993-1996. Nutritional Sciences Journal, 24(1), 59-80.

Wallengren, C., Friberg, F., \& Segesten, K. (2008). Like a shadow - on becoming a stroke victim's relative. Scandinavian Journal of Caring Sciences, 22(1), 48-55. doi: 10.1111/j.1471-6712.2006.00494.X

Yeh, P.-M., \& Chang, Y. (2011). Family carer reactions and their related factors among Taiwanese with hospitalized relatives. Journal of Advanced Nursing, 68(10), 2195-2206. doi: 10.1111/j.1365-2648.2011.05904.X 